\title{
Pre-Exercise Strategies: Effect of Yoga Poses on Various Intensities of Eccentric Exercise-Induced Pain and Functional Limitations
}

\author{
C. M. Sadacharan \\ School of Biological Sciences (SBS), University of New England (UNE), Biddeford, Maine, USA
}

\author{
CORRESPONDING AUTHOR: \\ Chakravarthy Marx Sadacharan \\ School of Biological Sciences \\ University of New England (UNE) \\ Biddeford, Maine-04005, USA \\ E-mail: csadacharan@une.edu
}

DOI:

10.32098/mltj.01.2021.11

LEVEL OF EVIDENCE: 2B

\begin{abstract}
SUMMARY
Background. Unaccustomed exercise can cause muscle damage, pain, and delayed onset of muscle soreness (DOMS) that may lead to temporary functional impairments. Performing yoga poses have many health benefits like it, increases flexibility, reduces anxiety and stress, and pain relief. The present study aimed to measure the effects of yoga poses performed before the various intensities of elbow and knee eccentric contraction (ECC) exercise protocol with a full (FROM) and partial range of motion (PROM).

Methods. Thirty participants aged between 18 and 30 years performed yoga before ECC protocols (weeks 3, 5, 7, 9) and ECC protocols without yoga poses (weeks 2, 4, 6, 8) on elbow and knee muscles $(5 \times 10$ repetitions each session) using their $25 \% / 35 \%$ of maximal voluntary isometric contraction (MVIC). The subjective and objective assessments were carried out (activities of daily living (ADL), pain (visual analog scale (VAS), pain pressure threshold (PPT)), and MVIC.

Results. The ECC exercise with FROM without yoga showed moderate pain (0-3.5) in ADL, VAS, and PPT for elbow extensors and knee flexors and showed a statistically significant difference $(\mathrm{p} \leq 0.05)$ compared to PROM without yoga ECC exercise protocol. ECC exercise performed with FROM without yoga induced a higher degree of DOMS, pain, ADL limitations than PROM without yoga. Yoga before PROM ECC protocol showed less pain, DOMS in ADL (pulling a heavy), VAS, and PPT for elbow flexors and knee extensors. The overall percentage of increased MVIC value at the end of the study was between 32.60 and $45.02 \%$.

Conclusions. Upper extremity (extensors) muscles are more prone for DOMS and pain when compared to lower extremity muscles. Yoga stretching poses before eccentric exercise protocol may have an effect in reducing DOMS and pain.
\end{abstract}

\section{KEY WORDS}

Exercise; pain; functional limitations; muscle soreness; yoga.

\section{INTRODUCTION}

Post-exercise muscle soreness or pain, also known as delayed onset of muscle soreness (DOMS) develops within 24 hours of exercise $(1,2)$. Exercise involving eccentric (ECC) muscle contractions results in greater muscle tissue damage or injury than does concentric $(\mathrm{CON})$ exercise $(1,3)$. The magnitude of muscle damage is influenced by intensity, repetition, velocity, and range of motion (ROM; muscle contraction length) $(4,5)$. Individuals can experience DOMS in varying degrees depending upon their level of fitness and the intensity and type of exercise performed. For example, a sedentary individual may experience a severe amount of DOMS than an athlete for a given set of exercise intensity. In severe DOMS, individuals may feel pain, temporary discomfort, and difficulty in performing activities of daily living (ADLs). Several researchers have reported various interventions to treat DOMS (1,5-7). Yoga popularity has grown tremendously in the past several years to treat many diseases. Yoga involves a process of physical and mental training including breath control, simple meditation, and the adoption of specific bodily postures. Yoga is widely practiced for health and relaxation. Performing yoga poses have tremendous health benefits like relief pain (8-12) and improve flexibility and balance (13), muscle strength (14, 15), skeletal muscle oxygen uptake (16), and lung function 
(17) and many more. Yoga poses are recommended along with workout sessions at the gym. Yoga can be performed before or after the exercise protocol depends upon intensity, type, and repetitions of the exercise protocol, and the types of yoga poses. In the published literature search, we could find a single study showing the effect of yoga poses on ECC exercise induced DOMS. A study by Boyle et al. (18) showed that yoga training and a single bout of yoga appear to attenuate peak DOMS in women following a bout of bench-stepping eccentric exercise.

We hypothesized that the intensity of functional impairment, DOMS, pain, and muscle strength would be lower when yoga poses performed before ECC exercise protocol, compared with those without yoga poses, but performed only ECC exercise protocol. We choose specific yoga poses that involve elbow and knee muscle groups to see the effect of yoga on the ECC exercise protocol with full and partial range of motion.

The purpose of the present study was to determine the effects of yoga poses performed before the various intensities of the elbow and knee ECC exercise protocol with different range of motion (ROM).

\section{METHODS}

\section{Study Design}

A cross-sectional study with an experimental design was conducted to investigate the effects of yoga poses performed before the various intensities of elbow and knee exercise protocol with different ROM.

\section{Subjects}

Thirty untrained active healthy individuals (12 men and 18 women) were recruited after the screening procedure at the University of New England (UNE) students aged between 18 and 30 years. The study has been approved by the authors' Institutional Review Board (UNE/IRB-20180508-004) and conducted in the Biology Research lab at Alfond Center for Health Sciences (ACHS) building and Campus Center Gym, UNE, Maine, USA. The study procedure was explained to all the participants and written informed consent was obtained prior to their participation in the research study. The author who involved in this study has followed the international ethical principles as well as the ethical standards of the Muscle, Ligaments and Tendons Journal (19).

\section{Procedures}

This study was carried out for a period of nine weeks that included: Week 1 - pre-test screening, the familiarization session, and MVIC calculation. Every week, the research participants were called for six days where only two days (day 1 and day 4) performed ECC exercises on elbow flexors, elbow extensors, knee flexors and knee extensors and remaining days 2, 3, 5 and 6, subjective (ADLs, Visual analog scale (VAS) and objective (Pain pressure threshold $(\mathrm{PPT})$ ), and muscle strength (MVIC)) assessments were carried out by the investigators. Weeks 2, 3, 4, and 5 elbow and knee ECC exercise protocol using their MVICs (25\% (week 2), yoga $+25 \%$ (week 3), 35\% (week 4) and yoga $+35 \%$ (week 5$)$ ) at a partial range of motion (PROM) $\left(60^{\circ}\right.$ of total ROM - an outer range of motion), subjective and objective assessments, and post ECC protocol MVIC calculation; weeks 6, 7, 8, and 9 - elbow and knee ECC exercise protocol using their MVICs (25\% (week 6), yoga $+25 \%$ (week 7), 35\% (week 8) and 35\% (week 9)) at the full range of motion (FROM), subjective and objective assessments, and post ECC protocol MVIC calculation (figure 1).

\section{Familiarization}

Week 1 - day 1 (visit 1): the investigator reviewed the study and the informed consent form with research participants. The study procedure including step by step to do maximal voluntary isometric contraction (MVIC) and eccentric (ECC) exercises in four of their muscle groups (elbow flexors, elbow extensors, knee flexors, and knee extensors), associated risks that may arise due to their participation, and the benefit of this research project were explained.

The five yoga poses were selected and demonstrated using yoga mats to all the research participants by the certified yoga instructors. The yoga poses were performed by the research participants before PROM and FROM ECC exercise protocol in weeks 3, 5, 7, and 9 (2 days per week). Yoga sessions were conducted as a group activity and repeated five times. All yoga sessions were started with a breathing exercise (6-8 times). A total duration of 20-30 mins was spent on each yoga session. The five yoga poses are:

a) child's pose - participants sat on the heels, then slowly brought their forehead down to rest in front of their knees. Rested the arms down alongside the body and took eight deep breaths.

b) thread the needle pose - participants sat on the heels and slid the right hand between the left hand and left knee. Slid the arm all the way out to the left so that the right shoulder and side of the head rested comfortably on the floor. Then, inhaled and reached the left hand up towards the ceiling. At first, explored the posture with the arm, found the place where the participant felt the deepest stretch, then stayed there and reached out through the fingers. Breathed and held for 6-8 breaths. To release: exhaled the palm back to 


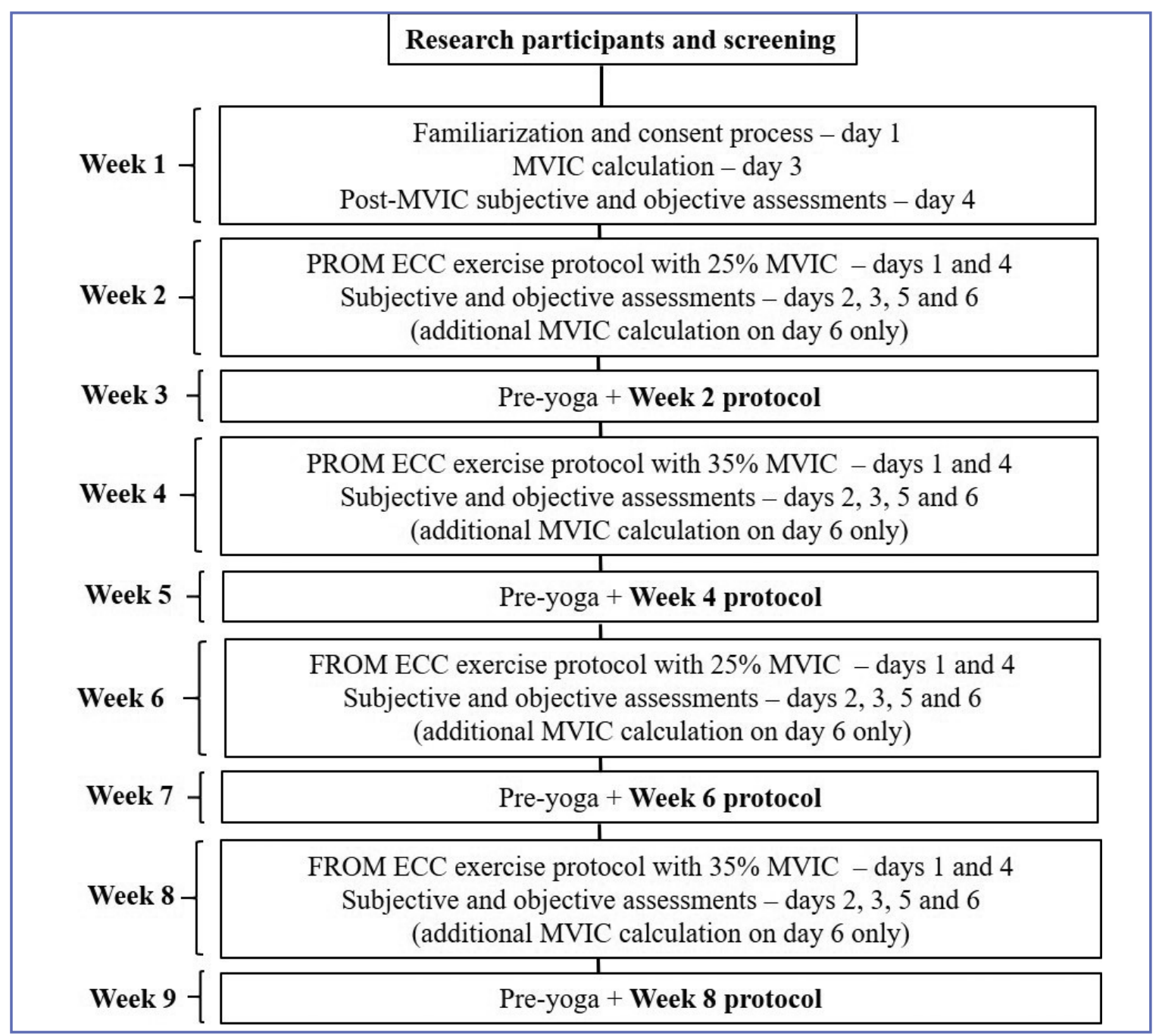

Figure 1. Simplified experimental design.

the floor and slowly inhaled back to sitting on the heels. The same was repeated on the other side.

c) eagle arms - participants stood tall in mountain pose. Wrapped one leg around the other, brought their bent arms out in front and wrapped their arms together in the opposite way, and slightly bent knees. Perched on a tree-like an eagle. Breathed and held for 6-8 breaths.

d) cow face pose - participants sat in a crossed-leg position, right leg over the left. Spread the legs as far apart as possible without bending their knees. The participant bent the left knee and placed the bottom of the left foot against the inner left thigh. Brought their left heel as close to the perineum as possible. Kept their left knee on the floor. After that, grasped the right foot with the left hand, and kept the foot on the floor, placed the heel of the right foot against the front-left portion of the left buttock. The right knee was directly on top of the left knee. Inhaled slowly through the nostrils and raised the right hand over the head and bent the right elbow. Reached behind the back with the left hand and clasped the fingers of both hands (forming an "s" shaped lock). Maintained the posture comfortably hold the inhaled breath. Later, exhaled slowly and then repeated the posture reversing the arms and legs. Breathed and held for 6-8 breaths.

e) hands overhead in wide-legged forward bend - from mountain pose, stepped their feet out wide and bent their upper body and clasped the hands together. Pretended that the participant's arms were the trunk of an elephant. Then, kept their hands overhead. Breathed and held for 6-8 breaths (figure 2). 


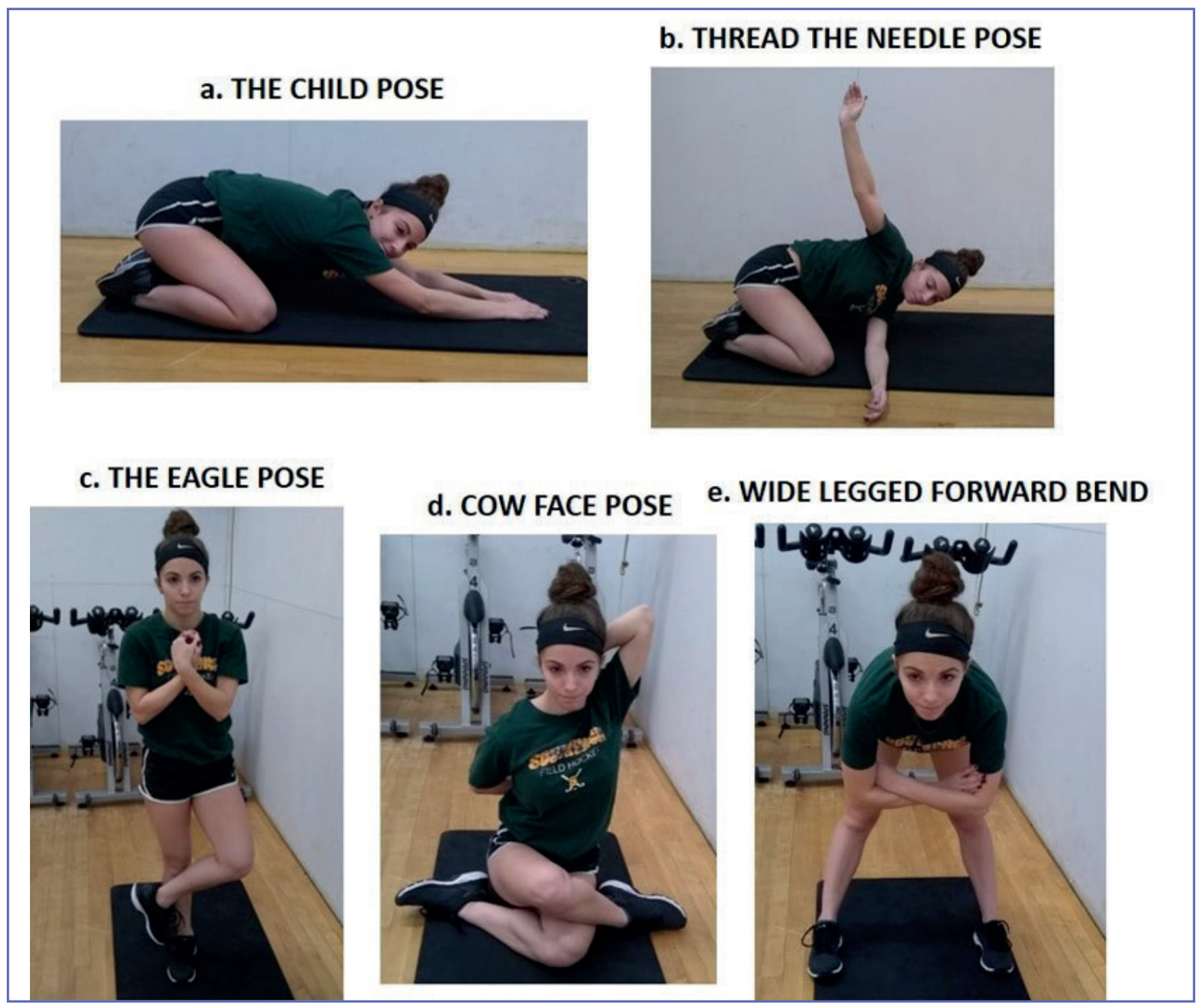

Figure 2. The yoga stretching poses were performed by the research participant.

The MVICs were performed on four selected muscles using a hand-held dynamometer (Lafayette Instrument, Manual Muscle Testing (MMT) Device, Pro-Health Care, USA) for 4 positions: 1) elbow flexion at 90 degrees; 2) elbow extension at 90 degrees; 3 ) knee flexion at 90 degrees; and 4) knee extension at 90 degrees. Depending on the visit, $25 \%$ or $35 \%$ of MVICs were calculated for the ECC exercise protocol.

Baseline delayed onset of muscle soreness (DOMS) assessment after MVIC testing

Week 1: after 24 hours of familiarization, the assessment of the ADL, DOMS, pain, and muscle strength were carried out.

\section{Subjective assessments}

A) health activities of daily living (ADL) difficulty scale - the following questions were asked. The pain scale ranged from 0 (no pain) to 10 (worst pain). For elbow: 1. Combing hair, 2. Eating with a fork or spoon, 3. Pulling a heavy object, 4. Using arm to rise from a chair, 5. Carrying an object above the shoulder, 6. Putting on shirt/coat, 7. Washing opposite armpit and back for knee: 1. Getting in and out of car, 2. Walking on the flat ground, 3. Ascending stairs, 4. Descending stairs, 5. Getting in and out of bed, 6. Bending to pick up from the floor. B) visual Analog Scale (VAS) - the level of muscle soreness was quantified using an 11 points VAS in which 0 indi- 
cated "no pain" and 10 represented "extreme pain". The participants were asked to mark the level of perceived soreness on the VAS when the elbow flexors, elbow extensors, knee flexors, and knee extensors are palpated in a circular motion by the investigator.

C) the delayed-onset of muscle soreness (DOMS) was assessed by asking the participants to perform the movements at the elbow and knee joints (flexion for extensors and extensors for flexors) and pain level was recorded based on the verbal response of the participants.

\section{Objective assessments}

Pressure Pain Threshold (PPT) - PPT is the minimum amount of force that can be applied to induce pain, that was measured using an electronic algometer (Baseline 60-pound Dolorimeter/Algometer Pain Threshold Meter, Pro-Health Care, USA). The VAS and PPT measurements helped us to compare the research participants' pain responses in the induced DOMS.

\section{Eccentric (ECC) Exercise Protocol with PROM and FROM}

The calculated elbow and knee flexors or extensors, 25\% or $35 \%$ of MVIC was attached to the research participant's wrist and ankle using Ultimately Fit Adjustable Ankle Weights (Hayneedle Company, USA) and facilitated the PROM and FROM ECC exercise protocols. The dynamic adjustable ROM controlled elbow and knee joint splints (T Scope ${ }^{\circledR}$ Elbow Premier (07254) and T Scope ${ }^{\circledR}$ Premier Post-Op Knee Brace (08814), BREG, USA) were used to limit the partial ROM $\left(60^{\circ}\right.$ of total ROM - the outer range of motion) while performing ECC protocols with PROM. These splints have locks to control the ROM so unexpected or uncontrolled movements at the elbow and knee of the subjects were prevented. We did not use any splint or external devices while performing ECC protocols with FROM (weeks 6-9) (figure 1).

\section{ECC Exercise Protocol}

Each week (week 2-9) - participants were introduced to ECC exercise on day 1 and 4 (5 sets of 10 repetitions for all four muscle groups) at PROM/FROM with calculated $25 \% / 35 \%$ of MVIC. Post- ECC Exercise assessment: day 2, 3, 5 and 6-After every ECC exercise protocol, the subjective and the objective assessments were carried out for 48 hours to see the functional impairment involving elbow and knee joints. The muscle strength (MVIC) was calculated for all four muscle groups at the end of each week.

\section{Statistical analysis}

Statistical analysis was carried out using GraphPad Prism software (version 8.2.0), San Diego, CA. The paired-sample t-test and repeated measures one-way ANOVA with Tukey's multiple comparison tests were carried out to compare the difference between pre-exercise, yoga with PROM ECC protocol, and yoga with FROM ECC protocol MVICs. Statistical significance was set at an alpha level at $\mathrm{p} \leq 0.05$.

\section{RESULTS}

In general, yoga before the PROM ECC protocol $(25 \%$ of MVIC) showed less pain, DOMS, and muscle strength when compared to FROM ECC protocol without yoga poses $(35 \%$ of MVIC). The pain level while pulling a heavy object (ADL) showed a moderate pain (pain level 3.7) at week 8 and mild pain (pain level 0.5) at week 3 and did show a statistically significant difference $(\mathrm{p} \leq 0.05)$ between them. Other ADL activities pain levels were less (pain level 0-0.5) and did not show any statistically significant difference between yoga prior to PROM and yoga prior to FROM ECC exercise weeks.

The pain level of VAS and DOMS assessments are shown in figure 3. Yoga prior to PROM ECC protocol (weeks 3 and 5) showed less pain on elbow extensors and knee flexors than yoga prior to FROM ECC protocol (weeks 7 and 9) in both VAS and DOMS assessments. Yoga prior to PROM ECC protocol (weeks 3 and 5) showed less pain on elbow extensors and knee flexors than PROM ECC protocol without yoga poses (weeks 2 and 4) in DOMS assessment and did show a statistically significant difference ( $\mathrm{p}$ $\leq 0.05$ ) between them. FROM ECC protocol without yoga poses (week 6 and 8) showed a moderate pain (pain level 2-4) on elbow extensors and knee flexors than yoga prior to FROM ECC protocol (week 7 and 9) in both VAS and DOMS assessments and did show a statistically significant difference $(\mathrm{p} \leq 0.05)$ between them.

PPT was compared between yoga prior to PROM and FROM exercise protocols (table I). The moderate PPT (pain level 4.2) was measured at the mid-belly of triceps brachii in the FROM ECC protocol without yoga poses (35\% of MVIC) at week 8 and mild PPT (pain level 6.6) was measured in the yoga prior to PROM ECC protocol (25\% of MVIC) at week 3. Mid-belly of the biceps brachii and mid-upper anterior thigh PPT values showed minimal pain and did not show any statistically significant difference between them. Both VAS and PPT results showed that elbow extensors with FROM ECC protocol at week 8 had moderate pain compared to elbow flexors, knee flexors, and knee extensors. 


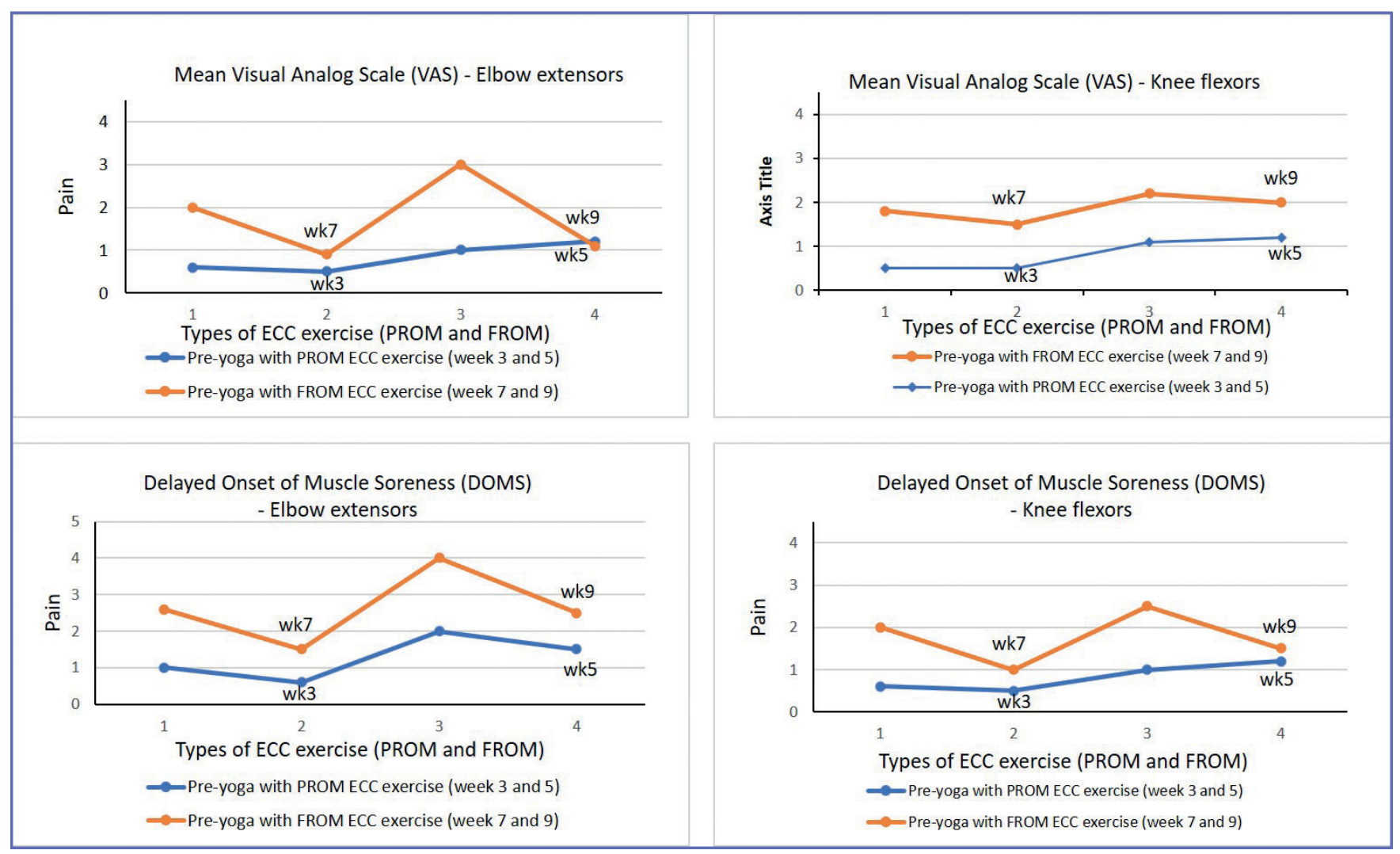

Figure 3. Visual analog scale (VAS) and delayed on set of muscle soreness (DOMS) comparison between pre-yoga with partial range of motion (PROM) and pre-yoga full range of motion (FROM) eccentric exercise protocols.

Table I. PPT comparison between yoga prior to PROM and yoga prior to FROM exercises using repeated measures one-way ANOVA with Tukey's multiple comparison test.

\begin{tabular}{lll}
\hline Weeks & EE & KF \\
\hline Week 2 vs week 3 & MD (sig.) & MD (sig.) \\
\hline Week 4 v week 5 & 0.42 (ns) & 0.42 (ns) \\
\hline Week 6 v week 7 & $1.252(* * * *)$ & $0.667(*)$ \\
\hline Week 8 v week 9 & $1.52(* * * *)$ & $1.12(* * * *)$ \\
\hline Week 3 vs week 5 & $1.987(* * * *)$ & $1.663(* * *)$ \\
\hline Week 5 vs week 9 & $0.12(\mathrm{~ns})$ & $0.12(\mathrm{~ns})$ \\
\hline Week 3 vs week 7 & $0.133(\mathrm{~ns})$ & $0.12(\mathrm{~ns})$ \\
\hline Week 5 vs week 9 & 0.12 (ns) & 0.13 (ns) \\
\hline
\end{tabular}

PPT - pain pressure threshold; PROM - partial range of motion; FROM - full range of motion; EE - elbow extensors; KF - knee flexors; week 2 - PROM exercise with 25\% of MVIC and no yoga; Week 3 - yoga then PROM exercise with 25\% of MVIC; week 4 - PROM exercise with $35 \%$ of MVIC and no yoga; week 5 - yoga then PROM exercise with 35\% of MVIC; week 6 - FROM exercise with 25\% of MVIC and no yoga; week 7 - yoga then FROM exercise with $25 \%$ of MVIC; Week 8 - yoga then FROM exercise with $35 \%$ of MVIC; week 9 - yoga then FROM exercise with $35 \%$ of MVIC; ns - not significance; * - significance; MD (sig.) - mean difference (significance). 
Yoga prior to PROM and FROM MVICs (weeks 3, 5, 7, 9) were compared and showed statistically significant differences $(p \leq 0.05)$ between them for all four muscle groups are shown in figure 4. The MVIC mean difference was increased with weeks. At the end of PROM ECC protocol (end of week 5), MVIC values were increased between 10.81 and $14.01 \%$ whereas after the FROM ECC protocol (end of week 9), MVIC values were increased between 17.63 and $25.21 \%$. The overall percentage of increased MVIC value at the end of the study was between 32.60 and $45.02 \%$.

\section{DISCUSSION}

The eccentric (ECC) exercises are a more effective way to exercise muscles than concentric exercises. Eccentric contractions work the muscle harder with less energy than concentric contractions. However, unaccustomed ECC may cause muscle damage, pain, DOMS that may lead to temporary functional impairments (20). Only temporary impairment (pain, soreness, reduced muscle performance) is associated with DOMS (21). No evidence exists to support the idea that DOMS is associated with long-term impairment or reduced muscle function. The level of ECC induced muscle pain and DOMS are influenced by intensity, repetition, and range of motion (ROM; muscle contraction length) (4).

The selected yoga poses were performed only prior to the ECC protocol. Should we do yoga before or after ECC protocol? This depends on many factors. For example, if the exercise depends more on flexibility than strength, then we would rather do it before the exercise and vice versa. The general belief is that yoga can be best performed after a strengthening exercise where we can focus all the muscle groups used and stretched. This may help to recover faster and can also aid in bringing the heart rate back down and relax. We can also do yoga as a warm-up. To take advantage of yoga before an exercise, make sure that we are doing dynamic stretches. Dynamic stretches will help to pump the heart and warm up the muscles, preparing for the exercise protocol. Even, yoga can be performed as a workout by continuing the poses for a longer time.

Performing certain yoga poses have many health benefits like increases flexibility, mobility, reduces anxiety and depression, mood, and stress, and, in many cases, all-natural pain relief (22). This study results showed that yoga

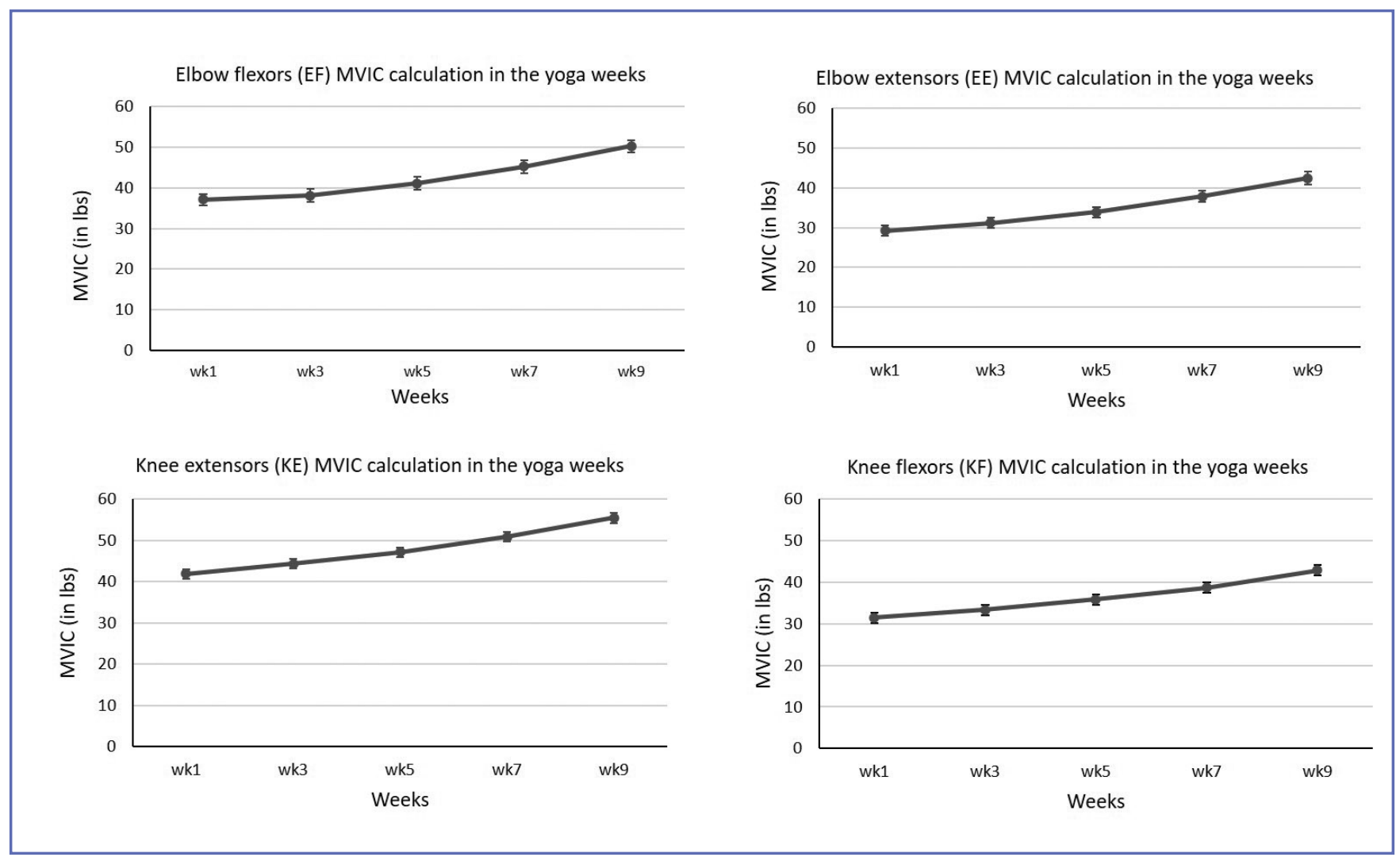

Figure 4. Maximal voluntary isometric contraction (MVIC) (muscle strength) on the muscles studied during the study process. 
prior to PROM ECC protocol on elbow and knee showed less pain, DOMS, and muscle strength when compared to FROM ECC protocol without yoga poses. The ECC protocols performed without yoga poses showed more pain and DOMS. The participants performed mild (25\% of MVIC) to moderate (35\% of MVIC) intensity ECC protocols and the yoga poses prior to ECC protocol were effective in relieving the muscle pain and DOMS.

The evidence suggests that joint ROM used during ECC exercise may also affect the muscle damage response $(23,24)$. De Giorgio et al. (25) study combined the yoga with back school intervention that lowered the anxiety, kinesiophobia and disability on the participants. Roya et al. (26) evaluated association of muscle fatigue with cervical ROM and concluded that cervical side bending was associated with changes in neck pain. The participants performed ECC protocol with PROM felt less pain and DOMS than ECC protocol with FROM. Greater the ROM ECC induces pain and DOMS whereas smaller the ROM ECC showed less pain and DOMS. In FROM ECC protocol the muscle is stretched that caused for the muscle damage, pain, and DOMS. In PROM ECC protocol the muscle is not stretched and minimal or no muscle damage, less pain, and DOMS. In this study, many factors like ROM, intensity, number of contractions, type of muscle contractions (ECC or CON), muscle groups exercised (flexors or extensors), and with or without yoga have influenced to determine the level of pain and DOMS. The participants performed ECC protocol with yoga poses perceived mild or no pain on elbow flexors and knee extensors. The participants performed ECC protocol without yoga poses perceived moderate pain on elbow extensors and knee flexors. Muscle strengths (MVIC) were increased in all the muscle groups studied. FROM ECC protocol increased the muscle strength compared to the PROM ECC protocol.

\section{REFERENCES}

1. Cheung K, Hume PA, Maxwell L. Delayed onset muscle soreness: treatment strategies and performance factors. Sports Med 2003;33:145-164.

2. Murase S, Terazawa E, Queme F, et al. Bradykinin and nerve growth factor play pivotal roles in muscular mechanical hyperalgesia after exercise (delayed onset muscle soreness). J Neurosci 2010;30:3752-3761.

3. Howatson G, van Someren KA. The prevention and treatment of exercise-induced muscle damage. Sports Med 2008;38:483-503.

4. Chen TC, Nosaka K, Sacco P. Intensity of eccentric exercise, shift of optimum angle, and the magnitude of repeated-bout effect. J Appl Physiol 2007;102:992-999.
In general, yoga poses involve many muscle groups of the body. Certain yoga poses work on specific muscle groups along with adjacent muscles. Child, thread the needle, eagle arms, cow face pose, and hands overhead in widelegged forward bend poses were performed in the study for 20-30 mins. The selected yoga poses involved all the muscle groups studied (elbow and knee) in this study. One of the challenges was to distinguish the effect of yoga on elbow and knee muscle groups alone. Studied muscle groups were limited to elbow and knee joints. Participants did not perform yoga poses after the ECC protocol. These were the limitation of the study.

\section{CONCLUSIONS}

Yoga prior to ECC protocol reduces the pain and DOMS on the elbow flexors and knee extensors compared to elbow extensors and knee flexors. Upper extremity (extensors) muscles are more prone to DOMS and pain when compared to lower extremity muscles. Yoga stretching poses prior to eccentric exercise protocol may have an effect in reducing DOMS and pain.

\section{ACKNOWLEDGMENTS}

The author thanks the School of Biological Sciences, University of New England (UNE) for the start-up fund, and participants who allowed the publication of this study. The author also thanks Dr. Erin Hartigan, PT Department, WCHP, UNE, Portland, Maine, USA for her support.

\section{CONFLICT OF INTERESTS}

The author declares that he has no conflict of interests.

5. Connolly DA, McHugh MP, Padilla-Zakour OI, Carlson L, Sayers SP. Efficacy of a tart cherry juice blend in preventing the symptoms of muscle damage. Br J Sports Med 2006;40(8):679-683.

6. Herbert R, de Noronha M, Kamper S. Stretching to prevent or reduce muscle soreness after exercise. Cochrane Database Syst Rev 2011;7:CD004577.

7. Torres R, Ribeiro F, Alberto Duarte J, Cabri JMH. Evidence of the physiotherapeutic interventions used currently after exercise-induced muscle damage: systematic review and meta-analysis. Physical Therapy in Sport 2012;13(2):101-114.

8. Barnes PM, Bloom B, Nahin RL. Complementary and alternative medicine use among adults and children: United States. Natl Health Stat Report 2008;10:1-23. 
9. Lazaridou A, Koulouris A, Devine JK, et al. Impact of daily yoga-based exercise on pain, catastrophizing, and sleep amongst individuals with fibromyalgia. J Pain Res 2019;12:2915-2923.

10. Pasyar N, Barshan Tashnizi N, Mansouri P, Tahmasebi S. Effect of yoga exercise on the quality of life and upper extremity volume among women with breast cancer related lymphedema: A pilot study. Eur J Oncol Nurs 2019;42:103-109.

11. Schmid AA, Fruhauf CA, Sharp JL, Van Puymbroeck M, Bair MJ, Portz JD. Yoga for People With Chronic Pain in a Community-Based Setting: A Feasibility and Pilot RCT. J Evid Based Integr Med 2019;24:2515690X19863763.

12. Chang DG, Holt JA, Sklar M, Groessl EJ. Yoga as a treatment for chronic low back pain: A systematic review of the literature. J Orthop Rheumatol 2016;3(1):1-8.

13. Polsgrove MJ, Eggleston BM, Lockyer RJ. Impact of 10-weeks of yoga practice on flexibility and balance of college athletes. Int J Yoga 2016;9(1):27-34.

14. Gothe NP, McAuley E. Yoga Is as Good as Stretching-Strengthening Exercises in Improving Functional Fitness Outcomes: Results From a Randomized Controlled Trial. J Gerontol A Biol Sci Med Sci 2016;71(3):406-411.

15. Gupta SK, Aparna S. Effect of Yoga Ocular Exercises on Eye Fatigue. Int J Yoga 2020;13(1):76-79.

16. Ha MS, Baek YH, Kim JW, Kim DY. Effects of yoga exercise on maximum oxygen uptake, cortisol level, and creatine kinase myocardial bond activity in female patients with skeletal muscle pain syndrome. J Phys Ther Sci 2015;27(5):1451-1453.

17. Budhi RB, Payghan S, Deepeshwar S. Changes in Lung Function Measures Following Bhastrika Pranayama (Bellows Breath) and Running in Healthy Individuals. Int J Yoga 2019;12(3):233-239.

18. Boyle CA, Sayers SP, Jensen BE, Headley SA, Manos TM. The effects of yoga training and a single bout of yoga on delayed onset muscle soreness in the lower extremity. J Strength Cond Res 2004;18(4):723-729.

19. Padulo J, Oliva F, Frizziero A, Maffulli N. Muscles, Ligaments and Tendons Journal - Basic principles and recommendations in clinical and field Science Research: 2018 update. MLTJ 2018;8(3):305-307.

20. Hody S, Croisier JL, Bury T, Rogister B, Leprince P. Eccentric Muscle Contractions: Risks and Benefits. Front Physiol 2019;10:536.

21. Vila-Chã C, Hassanlouei H, Farina D, Falla D. Eccentric exercise and delayed onset muscle soreness of the quadriceps induce adjustments in agonist-antagonist activity, which are dependent on the motor task. Exp Brain Res 2012;216(3):385-395.

22. Choudhary A, Pathak A, Manickam P, et al. Effect of Yoga versus Light Exercise to Improve Well-Being and Promote Healthy Aging among Older Adults in Central India: A Study Protocol for a Randomized Controlled Trial. Geriatrics (Basel) 2019;4(4):64.

23. Chapman D, Newton M, McGuigan MR, Nosaka K. Effect of lengthening contraction velocity on muscle damage of the elbow flexors. Med Sci Sports Exerc 2008;40(5):926-933.

24. Fochi AG, Damas F, Berton R, et al. Greater eccentric exercise-induced muscle damage by large versus small range of motion with the same endpoint. Biol Sport 2016;33(3):285-289.

25. De Giorgio A, Padulo J, Kuvačić G. Effectiveness of yoga combined with back school program on anxiety, kinesiophobia and pain in people with non-specific chronic low back pain: a prospective randomized trial. MLTJ 2018;8(1):104-112.

26. Roya M, Gholam Reza O, Azadeh S, Reza H.M, Saeed T.M. Changes of joint position sense in responses to upper trapezius muscle fatigue in subclinical myofascial pain syndrome participants versus healthy control. MLTJ 2018;8(4),534-543. 